\title{
The Role of High Resolution Sonography in the Evaluation of Anterior Abdominal Wall Lesions
}

\author{
C. Arpita', C Ananda Reddy ${ }^{2}$ \\ ${ }^{1}$ Associate Professor, Department of Radiology, Viswabharathi Medical College, Kurnool, Andhra Pradesh, India, ${ }^{2}$ Professor \& Head, Department of Surgery, \\ Viswabharathi Medical College, Kurnool, Andhra Pradesh, India.
}

\section{Abstract}

Background: Clinical presentation of anterior abdominal wall pathologies are non-specific. Based on physical examination alone, it is often difficult to diagnose the specific anterior abdominal wall pathologies. The aims of the study were, to evaluate accuracy of the high-resolution sonography in the diagnosis of anterior abdominal wall pathologies. Subjects and Methods: All patients referred to the Department of Radiodiagnosis with the clinical manifestations of various anterior abdominal lesion in a period of 2 years, were subjected for the study. 50 cases were taken up for the study. All patients included in the study underwent anterior abdominal wall ultrasonography using 7.0-12.0 MHZ high frequency linear array transducer coupled with color doppler equipment. This was followed by pelvic scan using 3.5-5.0 MHZ transducer whenever required. Findings during surgery and histopathology reports were noted and compared with the sonographic features. Results: Our study showed high prevalence of anterior abdominal lesions in patients between the age group of 20-40 years which constituted $60 \%$ of all cases. Most common presentation was seen in females $(66 \%)$. Incisional hernia was the predominant anterior abdominal wall lesion constituting $44 \%$, followed by ventral hernias $14 \%$ lipomas and hematomas both were seen in $8 \%$ of cases. Least common was anterior abdominal wall sarcoma seen in $2 \%$ cases. Higher resolution sonography is an accurate method for assessment of anterior abdominal wall lesions. Sensitivity of $100 \%$, specificity of $75 \%$, PPV of $97.4 \%$, NPV of $100 \%$. In total diagnostic accuracy of high-resolution sonography was $97.6 \%$ in our study. Conclusion: High resolution sonography is an accurate diagnostic imaging modality in anterior abdominal wall lesions. It is also highly sensitive in differentiating hernias from cystic or solid abdominal wall lesions.

Keywords: High Resolution; Ultrasonography; Anterior Abdominal Wall; Incisional Hernia; Ventral Hernia; Desmoid Tumor.

Corresponding Author: Dr. C Ananda Reddy, Professor \& Head, Department of Surgery, Viswabharathi Medical College, Kurnool, Andhra Pradesh, India.

Received: October 2019

Accepted: October 2019

\section{Introduction}

Abdominal wall lesions often mimic intra-abdominal conditions and frequently present as palpable masses. This is more common with patients who have a thick abdominal wall with a large layer of fat. Pathologic processes that mayinvolve the abdominal wall occasionally raise diagnostic challenges because of the low specificity of physical findings. The most common situation when a sonographic examination of the abdominal wall is needed is when there is a doubt about a palpable abdominal mass to decide whether it is in the abdominal wall or inside the abdominal cavity. Sometimes a clinically suspected intraabdominal mass proves to be in the wall, and sometimes an abdominal wall lesion is seen as an incidental finding on abdominal sonography performed for some other reason. Often patients with chronic abdominal pain need an examination of the abdominal wall, especially when a positive Carnett sign suggests the cause of pain to be in the abdominal wall. ${ }^{[1,2]}$

Pathology of the anterior abdominal wall is a challenge not only for the clinicians but also for the radiologist due to availability of wide range of imaging modalities for the evaluation of abdominal wall pathology ranging from plain X-ray abdomen to high resolution USG, CT scan, and magnetic resonance imaging.

With the introduction of high-frequency, high-resolution probes, detailed examination and recognition of different layers of the abdominal wall is now possible on USG examinations. A high-resolution examination can decide whether an abnormality is in the abdominal wall or inside the abdominal cavity. Physical findings in abdominal wall pathologies have low specificity and often a clinically suspected intra-abdominal lump proves to be in the abdominal wall. Typically when Carnett's sign is positive, a USG examination of the abdominal wall is advised.

High resolution USG due to its cost effectiveness, easily availability, lack of non-ionizing radiation and good accuracy rate makes it a supreme imaging modality in the evaluation of abdominal wall pathology.

There are wide range of pathologies affecting the anterior abdominal wall which range from simple fluid collections to hernias to complex neoplasms of the abdominal wall, hence early detection of these pathology with use of highresolution USG and other cross sectional imaging has 
revolutionized the treatment options for the surgeons. The present study also intended to assess the accuracy of high resolution sonography with high frequency linear array probe in diagnosis of anterior abdominal walllesions.

\section{Subjects and Methods}

Fifty cases of anterior abdominal wall lesions that were seen consecutively in the department of radiology during a 2 year study period from March, 2017- April 2019 in Viswabharathi Medical College, Kurnool (mainly from the department of surgery). These cases were evaluated using high resolution ultrasonography with high frequency linear array probe (7-12MHZ).

\section{Inclusion Criteria}

1. All cases with clinical manifestations of anterior abdominal wall lesions.

2. Cases of all age groups.

Exclusion criteria:

1. All cases with acute abdomen.

\section{Method of collection:}

All patients included in the study underwent anterior abdominal wall ultrasonographyusing7.0-12.0MHZ high frequency line ararraytransducercoupled with color doppler equipment (Esaote My Lab 50). This was followed by pelvic scan using 3.5-5.0 MHZ transducer whenever required.

All cases were critically evaluated using high resolution sonography (7-12 MHZ) with color doppler followed by surgical or pathological confirmation whenever needed.

Findings during surgery and histopathology reports were noted and compared with the sonographic features for assessing accuracy of high resolution sonography.

\section{Method of examination:}

All cases referred to the department of Radio-diagnosis with high degree of clinical suspicion of anterior abdominal wall lesions were evaluated using high resolution Ultrasonography with high frequency linear array probe. (7$12 \mathrm{MHZ}$ ).

\section{Stastical Methods:}

Diagnostic accuracy of anterior abdominal wall lesion using high resolution ultrasonography and colour doppler was determined by comparing with operative and histopathological findings, by performing diagnostic validity tests like sensitivity, specificity and predictive values.

\section{Results}

Our study showed high prevalence of anterior abdominal wall lesions in patients between the age group of 20-40 yrs. i.e. as many as 30 cases out of 50 cases (60\%) [Table 1]. Among 50 clinical suspected anterior abdominal wall lesions, most common indication for higher solution sonography was incisional hernia, followed by anterior abdominal wall lump which is relatively a non-specific clinical diagnosis, which later turned out be different lesions on high resolution sonography as well as histopathology.

Among 50 various clinically suspected anterior abdominal wall lesions which were subjected for high resolution sonography most commonly and accurately detected lesions of anterior abdominal wall was incisional hernia i.e. as many as 22 cases out of 50cases (44\%) followed by ventral hernias i.e. 7 case out of 50 cases (14\%) [Table 2].

Histopathological / post-operative findings were correlated in majority of the cases i.e. in 42 cases out of 50 cases whenever required. Cases diagnosed on ultrasonography which were subjected for histopathology / post-operative findings correlated accurately with our sonographic diagnosis in all cases with $97.6 \%$ accuracy [Table 3].

Table 1: Age wise distribution of anterior abdominal Wall lesions

\begin{tabular}{|l|l|l|l|}
\hline Sl. No. & Age Group & No. of Cases & Total \% \\
\hline 1 & $<20$ yrs. & 3 & $6 \%$ \\
\hline 2 & $20-40$ Yrs. & 30 & $60 \%$ \\
\hline 3 & $>40$ yrs. & 17 & $34 \%$ \\
\hline
\end{tabular}

Table 2: Various high resolution sonography of Anterior Abdominal Wall lesions

\begin{tabular}{|l|l|l|}
\hline Ultrasound Diagnosis & No. & $\%$ \\
\hline Lesions & 22 & 44 \\
\hline Incisional hernias & 7 & 14 \\
\hline Ventral hernias & 4 & 8 \\
\hline Anterior abdominal wall lipoma & 4 & 8 \\
\hline Anterior abdominal wall haematoma & 2 & 4 \\
\hline Desmoid tumour & 2 & 4 \\
\hline Dermoid cyst. & 1 & 2 \\
\hline Post operative seroma & 1 & 2 \\
\hline External oblique pyomyositis. & 1 & 2 \\
\hline Neurofibroma & 1 & 2 \\
\hline Metastatic melanoma & 1 & 2 \\
\hline Resolving abscess & 1 & 2 \\
\hline Abdominal wall sarcoma & 3 & 6 \\
\hline Others &
\end{tabular}

Table 3: Various Histopathological / Post-Operative diagnosis of anterior abdominal wall lesions

\begin{tabular}{|l|l|l|}
\hline \multicolumn{3}{|l|}{ Histopathology/Post-Operative Findings } \\
\hline & No. & \% \\
\hline Bowel loops & 20 & 40 \\
\hline Not done & 8 & 16 \\
\hline Dermoid cyst & 2 & 4 \\
\hline Desmoid fibromatosis & 2 & 4 \\
\hline Linea alba defect & 2 & 4 \\
\hline Lipoma & 4 & 8 \\
\hline Metastatic melanoma & 1 & 2 \\
\hline Neurofibroma & 1 & 2 \\
\hline Omental fat / bowel loops noted & 5 & 10 \\
\hline Abscess & 1 & 2 \\
\hline Others & 4 & 8 \\
\hline Total & 50 & 100 \\
\hline
\end{tabular}

Table 4: Diagnostic validity of high-Resolution Sonography

\begin{tabular}{|l|l|l|l|}
\hline Test & Positive & Negative & Total \\
\hline Positive & 38 & 0 & 38 \\
\hline Negative & 1 & 3 & 4 \\
\hline & 39 & 3 & 42 \\
\hline
\end{tabular}

Sensitivity $=100 \%$, Specificity $=75 \%$, Positive predictive value (Efficacy) $=97.4 \%$, Negative predictive value $=100 \%$ \& Diagnostic accuracy $97.6 \%$ 


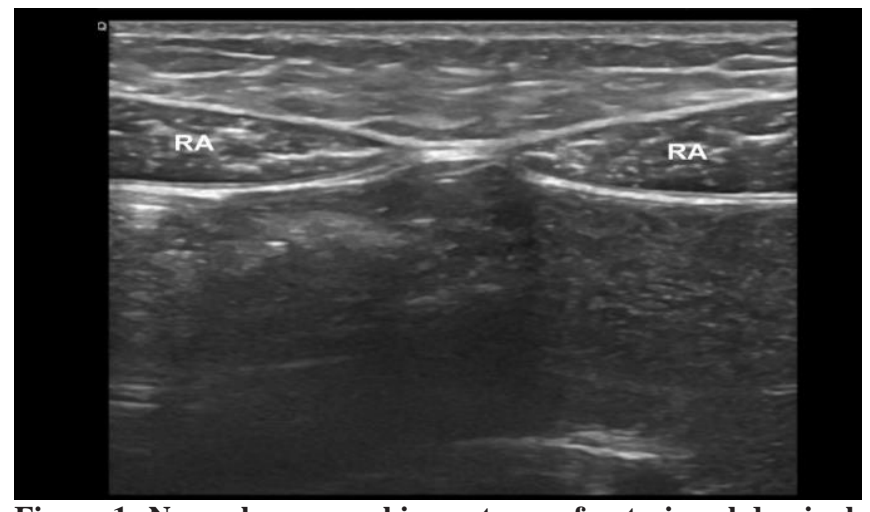

Figure 1: Normal sonographic anatomy of anterior abdominal wall

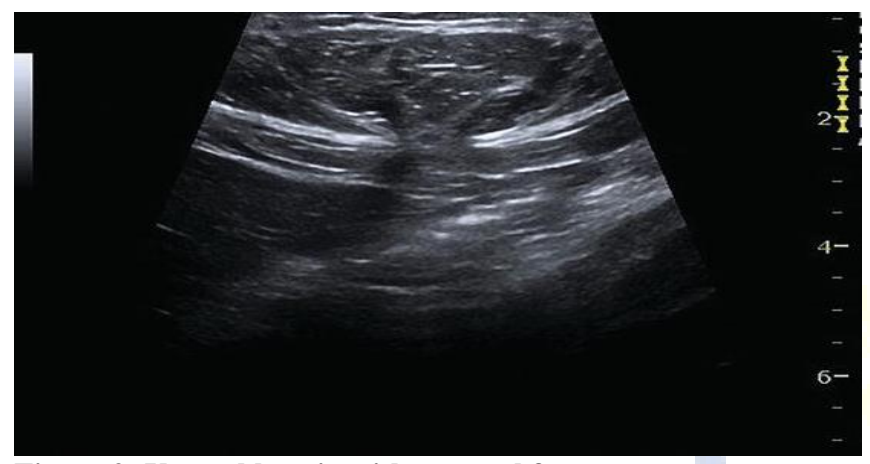

Figure 2: Ventral hernia with omental fat as content.
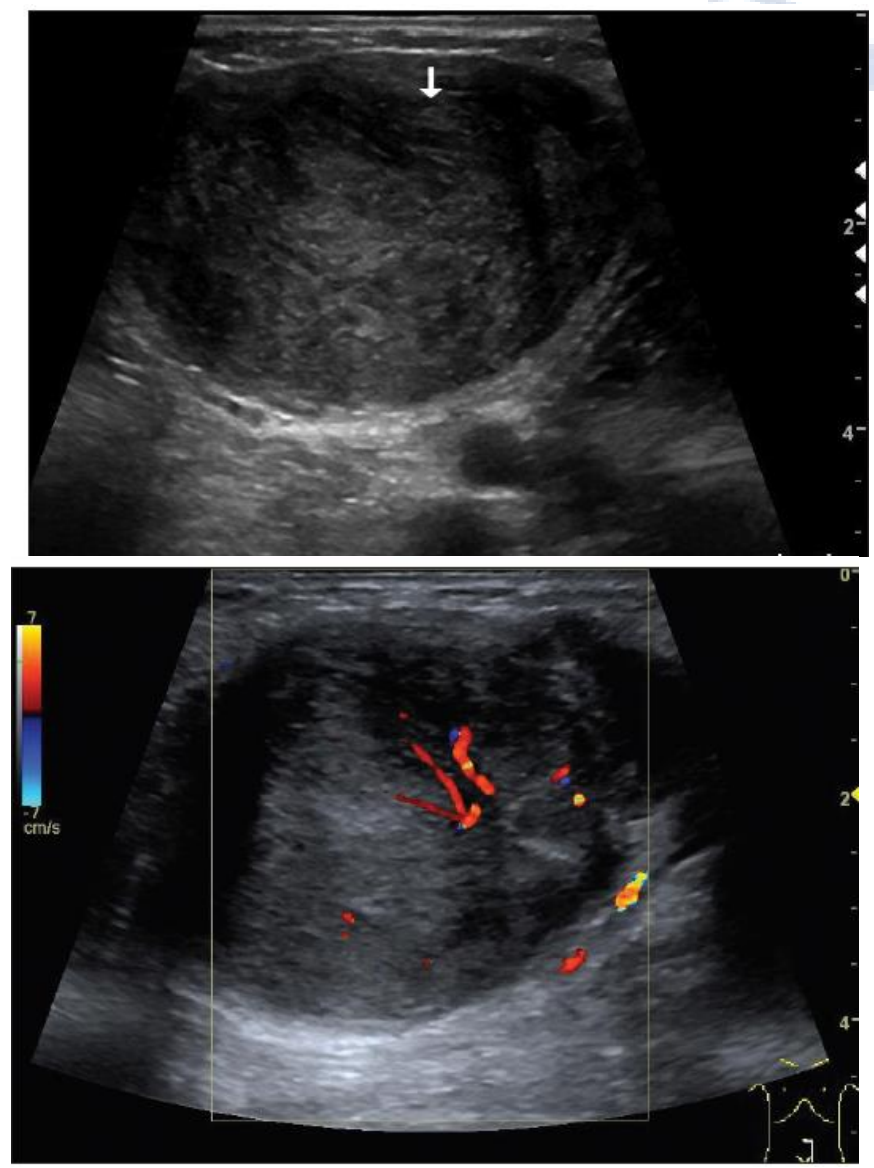

Figure 3: Rectus abdominis muscle desmoid tumor

\section{Discussion}

We collected 50 cases of anterior abdominal wall lesions of various types in a period of 2 years. Literature on this type of study is sparse in Indian literature and such study have not highlighted on incidence and prevalence of anterior abdominal wall lesions. Our study emphasized on diagnostic accuracy of high resolution sonography in detection of anterior abdominal wall lesions.

The time of onset of anterior abdominal wall lesions is well known entity in congenital anterior abdominal wall lesions like omphalocele, gastroschisis, cloacal exstrophy \& prune belly syndrome which can all be picked up during 2nd trimester antenatal sonography done during 18-24 weeks, hence age placed a paramount importance in diagnosis of anterior abdominal wall lesions, however we detected two cases of congenital anterior abdominal wall defect in antenatal scan, which are better done by using low frequency probe (3.5MHZ), but our study involves the use of high resolution, high frequency probe i.e (7-11 MHZ) hence fourth those cases were not included in our study.

We have categorized the age group of patients broadly into three groups i.e. $<20$ yrs, 20-40 yrs, and > 40 yrs. Our study showed a high prevalence of anterior abdominal wall lesions in the age group of 20-40 years (60\%), where in majority of cases were various types of incisional and ventral hernias. The higher prevalence of anterior abdominal wall lesions in the age group of 20-40 years probably explains the patients seeking medical/surgical attention more frequently than other age group due to various reasons.

Among 50 clinical suspected anterior abdominal wall lesions most common indication for high resolution sonography was incisional hernia i.e. 28 cases $(56 \%)$ out of 50 cases, followed by anterior abdominal lump i.e. 11 cases $(22 \%)$ out of 50 cases which is relatively a non-specific clinical diagnosis, which later turned out to be different lesions on high resolution sonography as well as histopathology which necessitates higher resolution sonography with high frequency probe which helps in characterization, nature of the lesion with a very high specificity than clinical findings and equal to or slightly less than that of histopathological and surgical findings.

Diagnosisofincisionalherniasandventralherniasonsonograph yisrelatively simple because sonography can readily detect the anterior abdominal wall defect, contents of the hernias, as well as the reducibility of hernias due to the real time imaging, with the application of Color Doppler sonography differentiation between strangulated and non-strangulated hernias can be made out. Anterior abdominal wall lipomas was the next common lesions detected in our study (4cases), all 4 cases were accurately detected on sonography with $100 \%$ accuracy, all 4 cases of anterior abdominal wall lipomas showed similar sonographic findings i.e. well defined round to ovoid iso to slightly hyperechoic echotexture as compared to the adjacent muscles along with a thin echogenic capsule.

Other lesions were desmoids tumors and dermoid cyst i.e. 2 
cases each (6.66\%). Sonographic diagnosis of both desmoid tumour and dermoid cyst was $100 \%$ accurate, where in desmoid tumour appeared as well defined hypoechoic lesions with increased vascularity on colour Doppler at the site of previous laparotomy scar.

Anterior abdominal wall lesions which were diagnosed on ultrasonography were subjected for histopathology / postoperative findings which correlated accurately with our sonographic diagnosis in all cases with $97.6 \%$ accuracy. We have shown that sonographic diagnostic accuracy parallel histopathological / post - operative findings.

Hernias are the most common abdominal wall lesions seen in sonographic practice. Depending on their location and cause, they are divided into different categories. With a high-frequency transducer, the fascial defect can be visualized underlying the hernia. Herniated bowel loops have a variable appearance depending on their air-fluid content and degree of obstruction. During the real-time examination, induction and reduction of a hernia can be observed.

A ventral hernia occurs typically where there is no muscle support along the linea alba in the midline in the epigastrium or periumbilical region. The defect can be easily made out in high frequency probe, and the defect size can also be measured. The hernia very often contains only fatty tissue but at times may be large and contain bowel loops also. ${ }^{[3,4]}$

An incisional hernia develops as a late complication of abdominal surgery. It also has been seen after laparoscopic procedures. Most incisional hernias will present with in the first year; however, some noticed by the patient and are incidentally detected on sonography or computed tomography. Sonography is very useful in ruling out a hernia at surgical sites and in monitoring the integrity of wire mesh implants. ${ }^{[5-7]}$

A spigelian hernia occurs because of a defect in the aponeurosis of the transverse abdomen is muscle and the rectus sheath. The most common site is the point where the line as emilunaris crosses the arcuate line. ${ }^{[8,9]}$ The hernias may sometimes extend laterally and present as a flank lump before the use of high resolution sonography, the diagnosis of spigelian hernia was missed in $50 \%$ of cases as per literature.
Depending on the content, namely, fluid, air-containing bowel, or fluid- containing bowel, inguinal hernias will have different appearances on sonography. Distended, adynamic bowel indicates obstructed loops. The inferior epigastric artery can be visualized on color Doppler sonography, and then differentiation of a direct or an indirect inguinal hernia is possible. ${ }^{[10,11]}$

\section{Conclusion}

High resolution sonography is an accurate diagnostic imaging modality in anterior abdominal wall lesions. It is also highly sensitive in differentiating hernias from cystic or solid abdominal wall lesions. High resolution sonography is also highly sensitive in differentiating intraabdominal from abdominal wall lesions. The advantages of high resolution sonography includes non-invasiveness, high accuracy, lack of ionizing radiation, simplicity, wide availability, cost effectiveness and repeatability.

\section{References}

1. Thomson WH, Dowes RF, Carter SS. Abdominal wall tenderness: a useful sign in chronic abdominal pain. Br J Surg 1991;78:223-225.

2. SuleimanS,JohnstonDE.Theabdominalwall:anoverlookedsourceofpain. Am Fam Physician 2001; 64:431-438.

3. ThomasJL,CunninghamJJ.Ultrasonicevaluationofventralherniasdisguis edas intra abdominal neoplasms. Arch Surg 1978;113:589-590.

4. Spangen L. Ultrasound as a diagnostic aid in ventral abdominal hernia. J Clin Ultrasound 1975;3:211-213.

5. Fischer JD, Turner FW. Abdominal incisional hernia: a ten year review. Can J Surg 1974;17:202-204.

6. Ellis H, Gajraj H, George CD. Incisional hernias: when do they occur? Br J Surg 1983;70:290-291

7. SpangenL.Spigelianhernia.ActaChirScandSupp11976;462:1-47.

8. Deitch EA, Engel JM. Spigelian hernia: an ultrasonic diagnosis. Arch Surg 1980;115:93

9. Korenkov M, Paul A, Troidl H. Color Duplex Sonography: diagnostic tool in the differentiationofinguinalhernias.JUltrasoundMed1999;18:565-568.

10. Riehl J, Schneider B, Sieberth HG. Femoral hernia: diagnosis with Bimage, duplex and color-coded Doppler ultrasound [in German]. Ultraschall Med 1995; 16:145-147.

11. Truong S ,Pfingstn FP, Dreuw B , Schumpelick V. Value of sonography in diagnosis of uncertain lesions of the abdominal wall and inguinal region.Chirurg1993;64:468-75.

Copyright: () the author(s), 2019. It is an open-access article distributed under the terms of the Creative Commons Attribution License (CC BY 4.0), which permits authors to retain ownership of the copyright for their content, and allow anyone to download, reuse, reprint, modify, distribute and/or copy the content as long as the original authors and source are cited.

How to cite this article: Arpita C, Reddy CA. The Role of High Resolution Sonography in the Evaluation of Anterior Abdominal Wall Lesions. Asian J. Med. Res. 2019;8(4):RD01-RD04.

DOI: dx.doi.org/10.21276/ajmr.2019.8.4.RD1

Source of Support: Nil, Conflict of Interest: None declared. 